\title{
Remodeling gut microbiota by Streptococcus thermophilus 19 attenuates inflammation in
}

2

3 Fu Han $^{* a}$, Yijie Zhang ${ }^{\text {ab }}$, Xuekang Yang ${ }^{* a}$, Zhuoqun Fang $^{\mathrm{a}}$, Shichao Han ${ }^{\mathrm{a}}$, Xiaoqiang Li ${ }^{\mathrm{a}}$, Weixia

$4 \mathrm{Cai}^{\mathrm{a}}$, Dan Xiao ${ }^{\mathrm{a}}$, Jiaqi Liu ${ }^{\mathrm{a}}$, Wanfu Zhang ${ }^{\mathrm{a}}$, Gaofeng Wu ${ }^{\text {a\# }} \&$ Dahai $\mathrm{Hu}^{\text {a\# }}$

5 a Department of Burns and Cutaneous Surgery, Xijing Hospital, Fourth Military Medical

\section{septic mice}

\author{
University, Xi'an, Shaanxi Province, 710032, China
}

b Key Laboratory of Tissue Repair and Regeneration of PLA and Beijing Key Research

Laboratory of Skin Injury, Repair and Regeneration, First Hospital Affiliated to General Hospital

$$
\text { of PLA, Beijing, 100048, China }
$$

Running title: Streptococcus thermophilus 19 attenuates inflammation in septic mice

\# Address correspondence to Gaofeng Wu, 15293136005@163.com and Dahai Hu, hudahaidoc@163.com.

*Fu Han, Yijie Zhang and Xuekang Yang contributed equally to this work

\section{Abstract}

Sepsis is a life-threatening organ dysfunction caused by a dysregulated host response to infection and is the leading cause of death in burn patients. Streptococcus thermophilus 19 is a highly effective probiotic, with well-studied health benefits, but its role in protecting viscera against injury caused by sepsis and the underlying mechanism is poorly understood. The goal of this study was to evaluate protection potency of S. thermophilus against inflammation in mice and evaluate the influence of sepsis and S. thermophilus on microbial community. We tested the utility of S. thermophilus 19 in attenuating inflammation in vitro and vivo of LPS-induced sepsis 
mouse model. We also evaluated the influence of sepsis and S. thermophilus on microbial

community. In vitro, S. thermophilus 19 decrease the expression of inflammatory factors.

24 Additionally, in a lipopolysaccharide-induced septic mouse model, mice administered the

25 probiotic 19 was highly resistant to Lps and exhibited decreased expression of inflammatory

26 factors compared to Lps-treated control mice. A MiSeq-based sequence analysis revealed that gut

27 microbiota alterations in mice intraperitoneally injected with $1 \mathrm{mg} / \mathrm{ml}$ LPS were mitigated by the

28 administration of oral probiotics 19. Together these findings indicate that S. thermophilus 19 may

29 be a new avenue for interventions against inflammation caused by sepsis and other systemic

Importance Sepsis is life-threatening organ dysfunction which is the leading cause of death in

41 inflammation, S. thermophilus 19, and investigating the therapeutic effect and mechanism of S. 
caused by sepsis and other systemic inflammatory diseases.

\section{$44 \quad$ Key words}

45 Sepsis, inflammation, Probiotics, Microbiome

\section{Introduction}

47 Sepsis is a life-threatening organ dysfunction caused by a dysregulated host response to infection needed.

The gut microbiota is a complex ecosystem consisting of trillions of bacteria that live in the digestive tracts of humans and other animals (3). Growing evidence supports the key role of a healthy gut microbiota in promoting and maintaining a balanced immune response and in the establishment of the gut barrier immediately after birth $(4,5)$. Moreover, a dysbiotic state of the gut microbiota can lead to dysregulation of various processes, which can in turn contribute to the development of autoimmune conditions (6). For instance, the presence or overabundance of specific types of bacteria may contribute to inflammatory disorders such as IBD (6). Additionally, metabolites from certain members of the gut flora may influence host signaling pathways, contributing to disorders such as colon cancer and obesity. Sepsis is an extreme response to inflammation that has profound effects on all parts of the body. For decades, the gut has been 
64 has a protective role during systemic inflammation. Thus, we hypothesized that intestinal

bacteria play an important role in sepsis since the gut microbiota is associated with many diseases.

Probiotics are live microbes that have beneficial effects on human and animal health when ingested in sufficient amounts (8). Probiotics play an important role in maintaining the normal microbiota composition and have been used to treat or prevent a number of gut health disorders, such as irritable bowel syndrome, hypercholesterolemia, gastritis, gut infection, parasitic infestation, hypersensitivity (including food allergies), and even certain types of cancers (e.g., colorectal cancer) $(9,10)$. The use of microbes as probiotics also hold potential for oral health in preventing and treating oral infections, dental plaque-related diseases, periodontal diseases and halitosis. Furthermore, probiotics can alleviate inflammation associated with some human diseases by promoting changes in the gut microbiota composition $(11,12)$. Streptococcus thermophilus is a highly effective probiotic that has well studied health benefits, including the production of antibiotics that prevent infections from pneumonia-causing microbes and $\mathrm{C}$. difficile and can help to prevent ulcers (13-15).

79 In this study, we used a coculture system (probiotics and RAW264.7 cells) to assess the ability of probiotics to decrease the expression of inflammatory factors. We showed that Streptococcus thermophilus 19 can decrease the inflammation induced by Lps in RAW264.7 cells. Furthermore, we investigated the ability of S. thermophilus 19 to protect mice against Lps-induced inflammation and gut microbiota alterations when administered as a probiotic. We observed that the administration of S. thermophilus 19 as probiotics could alter the gut microbiota composition 
85 of untreated mice or mice with Lps-induced sepsis, with the symptoms of sepsis mitigated in the

86 latter group. Moreover, the levels of several inflammatory factors in various organs were

87 correlated to a diverse gut microbiota composition. We hypothesize that supplementation of diets with probiotics protects visceral organs by reducing inflammation through alterations in the gut microbiota after sepsis.

\section{Results}

Probiotics decrease the expression of inflammatory factors in vitro

92 To assess the influence of the assayed probiotics on the expression of inflammatory factors, we developed a co-culture system (probiotics and RAW264.7 cells). After incubating for 6 hours, total RNA was extracted and the expression of inflammatory factors was assessed via quantitative RT-PCR. The Lps treatment increased the expression of inflammatory factors compared to the untreated group. After co-culturing with probiotics, we observed a reduction in inflammatory factor expression, particularly when cells were incubated S. thermophilus 19 (Figure1). At the same time, we investigated the influence of S. thermophilus 19 on the cell viability after treatment 6 hours. Results showed that S. thermophilus 19 didn't affect the cell viability after co-culture 6 hours (Supplementary Figure1). Therefore, S. thermophilus 19 was chosen for further study.

103 At first, the influence of different doses of Lps on mice survival rate was investigated. All mice died when the concentration of Lps exceeded $2.5 \mathrm{mg} / \mathrm{kg}$, even in mice administered probiotics 
with $2 \mathrm{mg} / \mathrm{ml}$ Lps, whereas only $20 \%$ of mice administered the same Lps without probiotics survived (Figure 2A). All mice treated with $1 \mathrm{mg} / \mathrm{kg}$ of Lps survived (Figure 2A). So, $1 \mathrm{mg} / \mathrm{kg}$ Lps was chosen to investigate the influence of S. thermophilus 19 on gut microbiota and inflammation of sepsis. Mice treated with Lps lost approximately $10 \%$ of their body weight during the 48 hours after injection, while untreated mice did not lose weight (Supplementary Figure2A). Although all treated groups regained their baseline weight by the third day, mice treated with S. thermophilus 19 exhibited high rates of body weight recovery. Total food and water intake and the animal health conditions for all mice were recorded. The reason we recorded the total water and food is we keep one group of mice in a cage. Lps-treated mice with or without probiotics exhibited a reduction in total drinking water and rat chow intake (Supplementary Figure2B). Furthermore, mice treated with the probiotics alone also exhibited decreased water and rat chow intake (Supplementary Figure 2B). However, mice treated with S. thermophilus 19 alone showed no changes in body weight, although they exhibited lower drinking water and food intake (Supplementary Figure2A and 2B). The decrease in body weight of the Lps-treated mice could be explained by the Lps-induced inflammation causing a reduction in food and drinking water intake, while the probiotics could alleviate inflammation to promote the recovery in body weight.

We observed a 2 -fold increase in TNF- $\alpha$ expression and 2.5 -fold in IL-1 $\beta$ while it was reduced to that observed in the control group in mice administered probiotics (Figure 2B). In contrast, in mice treated with probiotics without Lps treatment, no significant effect on the serum levels of IL-1 $\beta$ and TNF- $\alpha$ were observed compared to the control group, demonstrating that the 
127 probiotics has no influence on the host in the absence of sepsis.

128 Next, inflammation state of the kidneys, small intestines, livers and lungs of each mouse after

129 Lps and probiotic treatment was investigated. Lps treatment dramatically increased the

130 expression of IL-1 $\beta$, IL-6 and TNF- $\alpha$ in all tissues while they were effectively rescued in the

131 mice treated with S. thermophilus 19 compared to the mice treated with Lps alone (Figure 2C).

132 However, the expression of TNF- $\alpha$, IL- 6 and IL-1 $\beta$ in the probiotic- -treated mice and control

133 mice did not significantly differ (Figure 2C). H\&E staining revealed that compared with the liver

134 sections in control group mice, significant congestion of veins and hepatocyte necrosis was

135 observed in the Lps-treated mice, and the loss of intact liver plates and hepatocyte vacuolization

136 was observed (Figure 2D). In pulmonary sections, drastic destruction of alveolar structures was

137 detected in the Lps-treated mice, and the effusion in alveoli in these mice was markedly more

138 severe than that observed in the control group mice. Furthermore, tissue infiltration by

139 inflammatory cells was substantially higher in Lps-treated mice than in the control group mice.

140 Co-treatment with probiotics resulted in the restoration of a close-to-normal appearance of liver

141 and lung tissues. Moreover, S. thermophilus 19 treatment alone did not affect the liver and lung

142 sections of mice (Figure 2D).

\section{Lps altered the gut microbiota structure of mice}

144 In the balance between gut microbiota and inflammation, deviations either way may cause

145 corresponding adjustments in the other. To test whether the gut microbiota of mice was altered

146 due to sepsis, we collected cecal feces of mice and assayed them via MiSeq sequencing to

147 determine the composition of gut microbiota. Mice treated with Lps exhibited decreases gut 
microbiota richness compared to the control group (Chao1 index $)(\mathrm{P}<0.05)$ while no difference

Figure $3 \mathrm{~A}$ and $3 \mathrm{~B})(\mathrm{P}<0.05)$. In contrast, higher abundances of bacteria from the genus

Flavonifractor were observed in the Lps-treated mice. Interestingly, 8 OTUs were specifically

present in the Lps-treatment group compared to the control group, while the control group also

\section{Probiotics intervention alters the gut microbiota of mice}

To investigate the effect of probiotics on the gut microbiota of mice, we sequenced the gut

The relative abundance of gut microbiota in control and Lps group was showed in Figure 4D. In 
Figure $3 \mathrm{~A}$ and 3B $(\mathrm{P}<0.05)$. Nine OTUs were specifically present in the group treated with $\mathrm{S}$.

171 (Supplementary Figure 4).

\section{Oral administration of Probiotics alleviated viscera damage via altering the gut microbiota}

We showed that probiotic intervention can attenuate the inflammation in septic mice (Figure 2).

Furthermore, we previously reported that probiotics can reduce the inflammation induced by $\mathrm{Cr}$

175 (VI) in mice through modifying the gut microbiota. Thus, we hypothesized that the protection of

viscera by the probiotic-induced attenuation of inflammation in septic mice is also associated 
190 Lps-treated mice and the control group mice. Mice treated with Lps and S. thermophilus 19

191 exhibited decreases gut microbiota richness compared to the control group (Chao1 index)

$192(\mathrm{P}<0.05)$ while no difference in diversity (Shannon index) between two groups was observed

193 (Supplementary Figure5A and 5B). The gut microbiota of mice treated with Lps and 19 clustered

194 differently from that of the control group mice (Supplementary Figure 5C). The change in the

195 microbiota composition between Lps+ S. thermophilus 19 and control groups is shown in

196 Supplementary Figure 3 (in details) $(\mathrm{P}<0.05)$ and Supplementary Figure 5D. Six specific OTUs

197 were identified in the LPS7 group mice and 9 were identified in the control group

198 (Supplementary Figure 4). Taken together, these results indicated that all the treatments altered

199 the composition of gut microbiota of the assayed mice. Although the composition of gut bacteria

200 in mice treated with probiotic and that of control group differed, the expression of

201 inflammation-associated factors in these mice did not significantly differ. We speculated that the

202 gut microbiota in these exhibited a healthy status, whereas the probiotic and Lps-treated mice

203 had a lower health status.

204 Overall, these data showed that Lps and probiotics significantly impacted the microbiota

205 composition of mice.

The function of gut microbiota was specifically altered after the administration oral

\section{7 probiotics}

208 Next, we used a Kruskal-Wallis/Wilcoxon rank-sum test to determine how the altered

209 community structure of the gut microbiota affects its function. Mice treated with Lps and S.

210 thermophilus 19 were decreased in both primary bile acid biosynthesis and secondary bile acid 
211 biosynthesis, which have proinflammatory properties compared to the Lps-treated mice

212 (Figure6). These data suggest a significantly decreased proinflammatory signature, as well as an

213 increased anti-inflammatory capacity of the gut microbiome in probiotic-treated mice. Taken

214 together, the probiotics were observed to reshape the gut microbiota with a distinct composition,

215 network topology and functionality.

216 Discussion

217 Sepsis is life-threatening organ dysfunction caused by a dysregulated host response to infection

218 and often causes multiple organ damage. S. thermophilus has been shown to be highly effective

219 probiotic strains with well-studied health benefits. However, the impact of S. thermophilus on

220 the gut microbiota composition, and its influence on the inflammation caused by Lps-induced

221 sepsis remains poorly understood. In this study, we utilized a MiSeq sequencing approach to

222 assess how S. thermophilus 19 modulate the host fecal microbiota and inflammatory response in

223 an Lps-induced mouse sepsis model. Our results showed that S. thermophilus 19 can decrease

224 the expression of inflammatory factors RAW264.7 cells treated with Lps. Moreover, we showed

225 that S. thermophilus 19 were able to protect viscera against damage induced by sepsis.

226 Furthermore, S. thermophilus 19 could alter the microbiota composition and restore homeostasis

227 of the gut microbiota disrupted by sepsis.

228 Inflammation and infection are frequently accompanied by an imbalance in the intestinal

229 microflora(16). A strong inflammatory response may then be mounted against microfloral

230 bacteria, leading to a perpetuation of the inflammation and gut barrier dysfunction(17). Sepsis is

231 life-threatening organ dysfunction caused by a dysregulated host response to infection, which is 
often causes a systemic inflammatory response. To assess the relationship between the gut 
decreased the level of inflammatory factors in an LPS-induced sepsis mouse model. In addition, the administration of S. thermophilus 19 did not trigger any inflammation or dysbiosis of the gut microbiota, suggesting that they could safely be used to treat sepsis with no obvious harmful side effects. Thus, together with previous results, these results suggest that $\mathrm{S}$. thermophilus 19 may be one alternative probiotics for use in sepsis intervention in the future.

It has now been recognized that alterations in gut microbiota composition and function appear to

be an important mechanism by which probiotics alleviate human disease. Our results showed that 
274

275

276

277

278

279

280

281

282

283

284

285

286

287

288

289

290

291

292

293

294

treat to not only sepsis but also other systemic inflammatory diseases (inflammatory bowel disease, systemic inflammatory arthritis, multiple sclerosis and so on). Collectively, the results of our study provide a conceptual framework to further text this hypothesis in humans to treat sepsis and other systemic inflammatory diseases.

\section{Materials and Methods}

\section{Bacteria and media}

L. plantarum TW1-1, Pediococcus acidilactici XS40, L. plantarum DS45, L. paracasei LZU-D2,

L. delbruckii, L. casei 18-10, Streptococcus thermophilus 19 were provided by Dr. Xusheng Guo (Lanzhou University, Lanzhou, China) which were isolated from yogurt. Bacterial strains were cultured in De Man, Rogosa, and Sharpe (MRS; Beijing Solarbio Science \& Technology, Beijing,

China) growth medium with exception of 19 and XS40, which were cultured in M17 growth medium (MRS; Beijing Solarbio Science \& Technology, Beijing, China) supplemented with 1\% lactose and MRS medium supplemented with $0.5 \%$ glucose, respectively. MRS and M17 agar medium (Beijing Solarbio Science \& Technology, Beijing, China) were used to determine the CFU of the assayed probiotic strains.

\section{In vitro evaluation of inflammatory factors induced by probiotics}

The commercial immortal mouse macrophage cell line RAW264.7 was obtained from the American Type Culture Collection and was grown in Dulbecco's Modified Eagle's Medium (DMEM; Gibco, Gaithersburg, MD) supplemented with 10\% heat-inactivated fetal bovine serum (FBS) under a humidified $10 \% \mathrm{CO}_{2}$ atmosphere at $37^{\circ} \mathrm{C}$. In order to investigate the influence of probiotics, the cells were cultured in 12 -well culture plates at $1 \times 10^{6}$ cells/well. The bacterial 
strains were grown in MRS or M17 medium overnight (16 h), after which the cultures were

diluted to an optical density (OD) of 0.3 , washed with phosphate-buffered saline (PBS; pH 7.4),

resuspended in PBS, and were used to infect the RAW264.7 cells at a multiplicity of infection

(MOI) of 1:100 (cells/bacteria). The plates were incubated for $6 \mathrm{~h}$ at $37^{\circ} \mathrm{C}$ under a $10 \% \mathrm{CO}_{2}$

atmosphere and samples were collected to assess the levels of inflammatory factors by qRT-PCR.

PBS without bacteria was used as negative control.

\section{Animals and sepsis model}

302 The 7-14-week-old BALB/c $\left(H-2 D^{d}\right)$ mice (average weight $\left.20 \mathrm{~g}\right)$ used in this study were

originally purchased from the Experimental Animal Center of The Fourth Military Medical

University and were bred in our facility under specific-pathogen-free conditions. All animals

were maintained under a $12 \mathrm{~h}$ light/dark cycle. In order to investigate the effect of Lps on the

survival rate, mice were administered different doses of lipopolysaccharide (Lps) by

intraperitoneal injection. To investigate the influence of probiotics on sepsis, mice were

administered $1 \mathrm{mg} / \mathrm{kg}$ lipopolysaccharide (Lps) by intraperitoneal injection, with a second dose

administered 4 days after the first injection. The details of the experimental design are shown in

310 Table 1. The names of the experimental groups were renamed because of sequencing

\section{Weight, water and food intake measurements and sampling}

315 Body weight, water and food intake, and stool appearance were documented for all groups of 
mice every other day throughout the experiment. After 1 week, livers, kidneys, lungs and small intestines were collected from each mouse and were divided into triplicate samples, with one stored in liquid nitrogen, a second stored in RNAiso Plus for RNA extraction, and the third was fixed in $4 \%(\mathrm{w} / \mathrm{v})$ paraformaldehyde at $4{ }^{\circ} \mathrm{C}$ for later histological analysis.

\section{Histology of different tissues}

321

322

After the animals were sacrificed, different tissue samples were collected. After fixation in $4 \%$ paraformaldehyde, tissue samples were embedded in paraffin and serially cut into 7-mm thick sections. Tissue slides were stained with hematoxylin and eosin (H\&E) for histological analysis.

\section{Microbial DNA extraction and Illumina MiSeq sequencing}

Microbial DNA was extracted from the samples using an E.Z.N.A. ${ }^{\circledR}$ Stool DNA Kit (Omega BioTek, Norcross, GA, USA) according to manufacturer's protocols, and the DNA samples were assessed via PCR with the universal 16S rRNA primers 27F/1492R in our own lab. The DNA concentration and integrity were determined by electrophoresis on $1 \%$ agarose gels containing ethidium bromide and spectrophotometrically using an EPOCH instrument (BioTek). After confirmation, the DNA was lyophilized and sent for Illumina MiSeq sequencing and data analysis.

The gut microbiota compositions of mice were assessed via Illumina MiSeq sequencing (Genergy Biotech) targeting the V3-V4 region of the bacterial 16S ribosomal RNA gene using the primers 341F (5'-CCTACGGGNGGCWGCAG-3') and 785R (5'-GACTACHVGGGTATCTAATCC-3'), with an eight-base barcode sequence unique to each sample. The amplicons were extracted from 2\% agarose gels and purified using an AxyPrep 
337

338

339

340

341

342

343

344

345

346

347

348

349

350

351

352

353

354

355

356

357

DNA Gel Extraction Kit (Axygen Biosciences, Union City, CA, USA) according to the

manufacturer's instructions and were subsequently quantified using a QuantiFluor ${ }^{\mathrm{TM}}-\mathrm{ST}$

instrument (Promega, USA). The purified amplicons were pooled in equimolar ratios and

paired-end sequenced $(2 \times 300)$ on an Illumina MiSeq platform according to standard protocols.

The raw reads were deposited at the NCBI Sequence Read Archive (SRA) database. Operational taxonomic units (OTUs) were clustered with a 97\% similarity cutoff using UPARSE (version 7.1

http://drive5.com/uparse/), and chimeric sequences were identified and removed using UCHIME.

The taxonomy of each 16S rRNA gene sequence was analyzed using RDP classifier

(http://rdp.cme.msu.edu/) against the SILVA (SSU123) 16S rRNA database using a confidence

threshold of $70 \%$. The taxonomy of each ITS gene sequence was analyzed using Unite classifier

(https://unite.ut.ee/index.php).

\section{Quantitative RT-PCR for inflammatory factor determination}

Total RNA was extracted from different tissues using RNAiso Plus (Takara, Dalian, China) and was subsequently reverse transcribed into cDNA using PrimeScript ${ }^{\mathrm{TM}}$ RT Kit (Takara, Dalian,

China) according to the manufacturer's protocol. The expression of inflammatory factor-related genes was analyzed using SYBR ${ }^{\circledR}$ PremixEx Taq ${ }^{\mathrm{TM}}$ II and the Bio-Rad CFX system. For real-time PCR, the reaction mixtures contained $1 \mu \mathrm{L}$ cDNA, $0.4 \mu \mathrm{L}$ of each primer $\left(10 \mathrm{mmol}^{-1}\right)$, $5 \mu \mathrm{L}$ of SYBR green PCR Master Mix, and distilled water to a final reaction volume of $10 \mu \mathrm{L}$.

The Taq DNA polymerase was activated at $95^{\circ} \mathrm{C}$ for $10 \mathrm{~min}$, followed by 40 cycles of $95^{\circ} \mathrm{C}$ for $15 \mathrm{~s}, 60^{\circ} \mathrm{C}$ for $30 \mathrm{~s}$, and $72^{\circ} \mathrm{C}$ for $30 \mathrm{~s}$. Quantitative RT-PCR data were normalized to the expression of the housekeeping gene $\beta$-actin using the $2^{-\Delta \mathrm{Ct}}$ method. Primers used in this study 
are shown in Table 2.

Quantification and statistical analysis

Graphpad Prism was used for graphical presentation and statistical analyses. Differences were considered statistically significant at $\mathrm{p}<0.05$, and data are presented as the means \pm SEM. The number of biological replicates (n) and the number of independent experiments are indicated in the figure legends. The Kruskal-Wallis/Wilcoxon rank-sum test was used to analyze the gut microbiota composition data for all the groups.

\section{Acknowledgements}

We thank Dr. Xusheng Guo (Lanzhou University) for his assistance for supply our strains and

(81530064, 81801913, 81601680 and 81701902).

\section{Author contributions}

G.W. and D.H. designed and supervised the study. F.H., Y.Z. and X.Y. performed experiments

\section{Competing interests}

377 The authors declare that they have no conflict of interests 


\section{References}

1. Bai X, He T, Liu Y, Zhang J, Li X, Shi J, Wang K, Han F, Zhang W, Zhang Y, Cai W, Hu D. 2018. Acetylation-dependent regulation of notch signaling in macrophages by sirt1 affects sepsis development. Front Immunol 9:762.

2. Manning J. 2018. Sepsis in the burn patient. Crit Care Nurs Clin North Am 30:423-430.

3. Glenwright AJ, Pothula KR, Bhamidimarri SP, Chorev DS, Basle A, Firbank SJ, Zheng H, Robinson CV, Winterhalter M, Kleinekathofer U, Bolam DN, van den Berg B. 2017. Structural basis for nutrient acquisition by dominant members of the human gut microbiota. Nature 541:407-411.

4. Hand TW. 2016. The role of the microbiota in shaping infectious immunity. Trends Immunol 37:647-658.

5. Maynard CL, Elson CO, Hatton RD, Weaver CT. 2012. Reciprocal interactions of the intestinal microbiota and immune system. Nature 489:231-41.

6. Clemente JC, Manasson J, Scher JU. 2018. The role of the gut microbiome in systemic inflammatory disease. BMJ 360:j5145.

7. Carrico CJ, Meakins JL, Marshall JC, Fry D, Maier RV. 1986. Multiple-organ-failure syndrome. Arch Surg 121:196-208.

8. Isolauri E, Kirjavainen PV, Salminen S. 2002. Probiotics: a role in the treatment of intestinal infection and inflammation? Gut 50 Suppl 3:III54-III59.

9. Mombaerts P, Mizoguchi E, Grusby MJ, Glimcher LH, Bhan AK, Tonegawa S. 1993. Spontaneous development of inflammatory bowel disease in T cell receptor mutant mice. 
Cell 75:274-82.

401 10. Doron S, Snydman DR. 2015. Risk and safety of probiotics. Clin Infect Dis 60 Suppl 2:S129-S134.

403 11. Wu G, Xiao X, Feng P, Xie F, Yu Z, Yuan W, Liu P, Li X. 2017. Gut remediation: a potential approach to reducing chromium accumulation using Lactobacillus plantarum TW1-1. Sci Rep 7:15000.

406

407

408

409

410

411

412

12. Hwang IY, Koh E, Wong A, March JC, Bentley WE, Lee YS, Chang MW. 2017. Engineered probiotic Escherichia coli can eliminate and prevent Pseudomonas aeruginosa gut infection in animal models. Nat Commun 8:15028.

13. Correa NB, Peret Filho LA, Penna FJ, Lima FM, Nicoli JR. 2005. A randomized formula controlled trial of Bifidobacterium lactis and Streptococcus thermophilus for prevention of antibiotic-associated diarrhea in infants. J Clin Gastroenterol 39:385-9.

14. Saavedra JM, Bauman NA, Oung I, Perman JA, Yolken RH. 1994. Feeding of Bifidobacterium bifidum and Streptococcus thermophilus to infants in hospital for prevention of diarrhoea and shedding of rotavirus. Lancet 344:1046-9.

15. Mater DD, Bretigny L, Firmesse O, Flores MJ, Mogenet A, Bresson JL, Corthier G. 2005. Streptococcus thermophilus and Lactobacillus delbrueckii subsp. bulgaricus survive gastrointestinal transit of healthy volunteers consuming yogurt. FEMS Microbiol Lett 250:185-7.

16. Khosravi A, Yanez A, Price JG, Chow A, Merad M, Goodridge HS, Mazmanian SK. 2014. Gut microbiota promote hematopoiesis to control bacterial infection. Cell Host Microbe 
15:374-81.

422 17. Schuijt TJ, Lankelma JM, Scicluna BP, de Sousa e Melo F, Roelofs JJ, de Boer JD, pneumococcal pneumonia. Gut 65:575-83.

18. Ojima M, Motooka D, Shimizu K, Gotoh K, Shintani A, Yoshiya K, Nakamura S, Ogura

19. Zaborin A, Smith D, Garfield K, Quensen J, Shakhsheer B, Kade M, Tirrell M, Tiedje J, critical illness. MBio 5:e01361-e14.

20. Pantoflickova D, Corthesy-Theulaz I, Dorta G, Stolte M, Isler P, Rochat F, Enslen M,

Blum AL. 2003. Favourable effect of regular intake of fermented milk containing

437 21. Xin J, Zeng D, Wang H, Ni X, Yi D, Pan K, Jing B. 2014. Preventing non-alcoholic fatty 
Guarner C, Balanzo J. 2002. Effect of Lactobacillus johnsonii La1 and antioxidants on 37:456-62.

23. Borges NA, Stenvinkel P, Bergman P, Qureshi AR, Lindholm B, Moraes C,

24. Sonnenburg JL, Backhed F. 2016. Diet-microbiota interactions as moderators of human metabolism. Nature 535:56-64.

451 25. Wang J, Tang H, Zhang C, Zhao Y, Derrien M, Rocher E, van-Hylckama Vlieg JE, 
463

464

465

466

467

468

469

470

471

472

473

474

475

476

477

478

479

480

481

482

483

Figure.1. The expression of inflammatory factors (IL-1 $\beta$, TNF- $\alpha$ and IL-6) in Lps-treated, probiotics-Lps treated and untreated RAW264.7 cells. Error bars represents SEM. Illustration represent the influence of $\mathrm{S}$. thermophilus 19 on inflammatory factors. $* \mathrm{P}<0.05, * * \mathrm{P}<0.01$.

Figure.2. Probiotics alleviate the inflammatory caused by Lps-induced sepsis. (A) Survival rates of mice with or without probiotics treatment after $48 \mathrm{~h}$ stimulation with different dose of Lps $(n=10)$. (B) Levels of IL-1 $\beta$ and TNF- $\alpha$ in blood were determined using commercial ELISA kits $(n=8)$. (C) Probiotics intervention resulted in decreased inflammation small intestine, lung, liver and kidney $(\mathrm{n}=8)$. Error bars represents SEM. (D) Hematoxylin and eosin staining of liver, and lung tissues from different groups. Sections were examined and photographed under a microscope.

Figure.3. Lps induce significant impact on microbiota composition. (A) (B) Fecal microbiota alfa diversity. (C) PLS_DA plot of fecal microbiota of Lps-treated or control mice. (D) The change of gut microbiota at phylum level.

Figure.4. S. thermophilus 19 induce significant impact on microbiota composition compared to control group mice. 7 represent S. thermophilus 19 (n=8). (A) (B) Fecal microbiota alfa diversity. (C) PLS_DA plot of fecal microbiota of LPS-treated mice with or without S. thermophilus 19 treatment. (D) The change of gut microbiota at phylum level.

Figure.5. S. thermophilus 19 induce significant impact on microbiota composition compared to Lps-treated mice. 7 represent S. thermophilus 19 (n=8). (A) (B) Fecal microbiota alfa diversity. (C) PLS_DA plot of fecal microbiota of LPS-treated mice with or without S. thermophilus 19 treatment. (D) The change of gut microbiota at phylum level. 
484 Figure.6. The presence of S. thermophilus 19 induces changes in gut microbiota function after

485 Lps treatment. Statistical comparison was performed by first testing normality using

486 Kruskal-Wallis/Wilcoxon rank-sum test. Error bars represents SEM.

487 Supplementary Figure1. The effect of S. thermophilus 19 on cell viability was detected by

488 CCK8 assay after co-culture 6hours. Error bars represents SEM.

489 Supplementary Figure2. The influence of S. thermophilus 19 and Lps on body weight, toal rat

490 chow and drinking water intake. (A) Body weight change and relative weight change

491 (n=8/group). (B) Total rat chow intake and drinking water.

492 Supplementary Figure3. The details of the change of gut microbiota at phylum (A) and genus

493 (B) level in different groups. Data with significant changes were showed in the figure $(\mathrm{P}<0.05)$.

494 Supplementary Figure4. Specific OTUS existed in different groups.

495 Supplementary Figure5.The gut microbiota composition between control group and

496

497

498

499

500

501

502

503 co-treatment (19 and Lps) group (n=8). (A) (B) Fecal microbiota alfa diversity. (C) PLS_DA plot

of fecal microbiota of Lps-treated mice with S. thermophilus 19 treatment and control group. (D)

The change of gut microbiota at phylum level. 
505

506

Table1 Experimental design

\begin{tabular}{ccc}
\hline Group & Treatment Groups(n=8) & Gavaging \\
\hline 1 & Control & PBS \\
2 & Lps only & PBS \\
3 & Lps+S. thermophilus 19 & PBS+S. thermophilus 19 \\
4 & S. thermophilus 19 only & PBS+S. thermophilus 19 \\
\hline
\end{tabular}

507 Lps: $1 \mathrm{mg} / \mathrm{ml}$; S. thermophilus 19: $1 \times 10^{9} \mathrm{CFU} / \mathrm{ml}$ once every other day in $0.3 \mathrm{ml} \mathrm{PBS}$. Mice received Lps( $\left.1 \mathrm{mg} / \mathrm{kg}\right)$

508 through intraperitoneal injection. Mice received PBS and 19 via gavage.

509 Table2 Primers used in this study

\begin{tabular}{cc}
\hline Primer & Sequence(5'-3') \\
\hline$\beta$-actin & GTACGCCAACACAGTGCTG/CGTCATACTCCTGCTTGCTG \\
IL-1 $\beta$ & GCTTCAGGCAGGCAGTATC/AGGATGGGCTCTTCTTCAAAG \\
TNF- $\alpha$ & AGAGCTACAAGAGGATCACCAGCAG/TCAGATTTACGGGTCAACTTCACAT \\
IL-6 & GAGGATACCACTCCCAACAGACC/ AAGTGCATCATCGTTGTTCATACA \\
\hline
\end{tabular}

510

511 


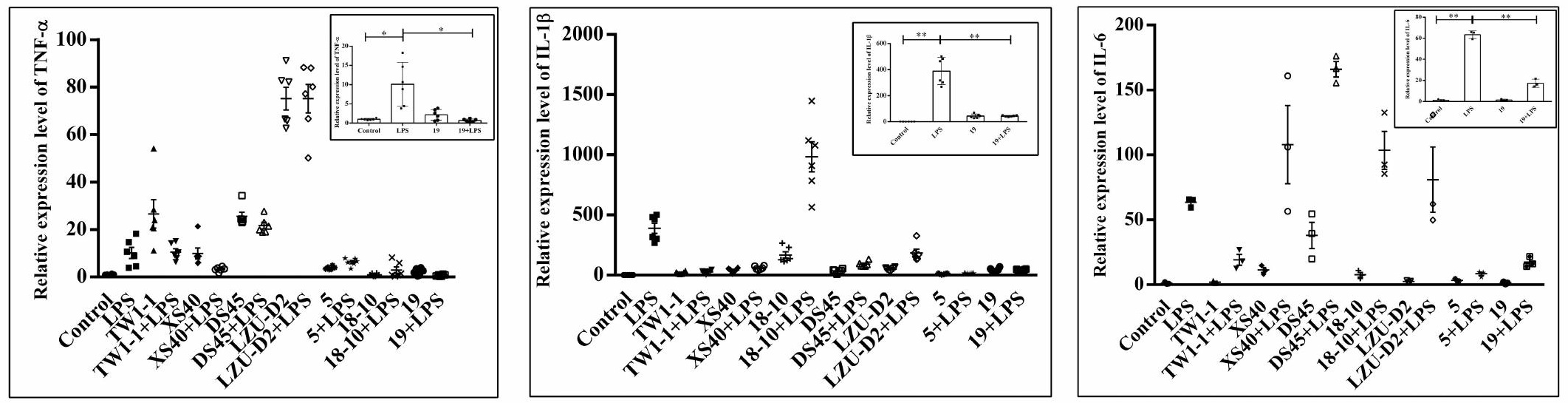




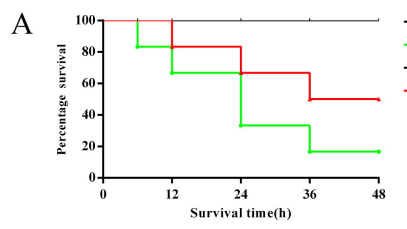

- LPS $2 \mathrm{mg} / \mathrm{ml}$
- LPS $1 \mathrm{mg} / \mathrm{ml}+\mathrm{S}$. thermophilus 19

$\underbrace{B}_{0}$
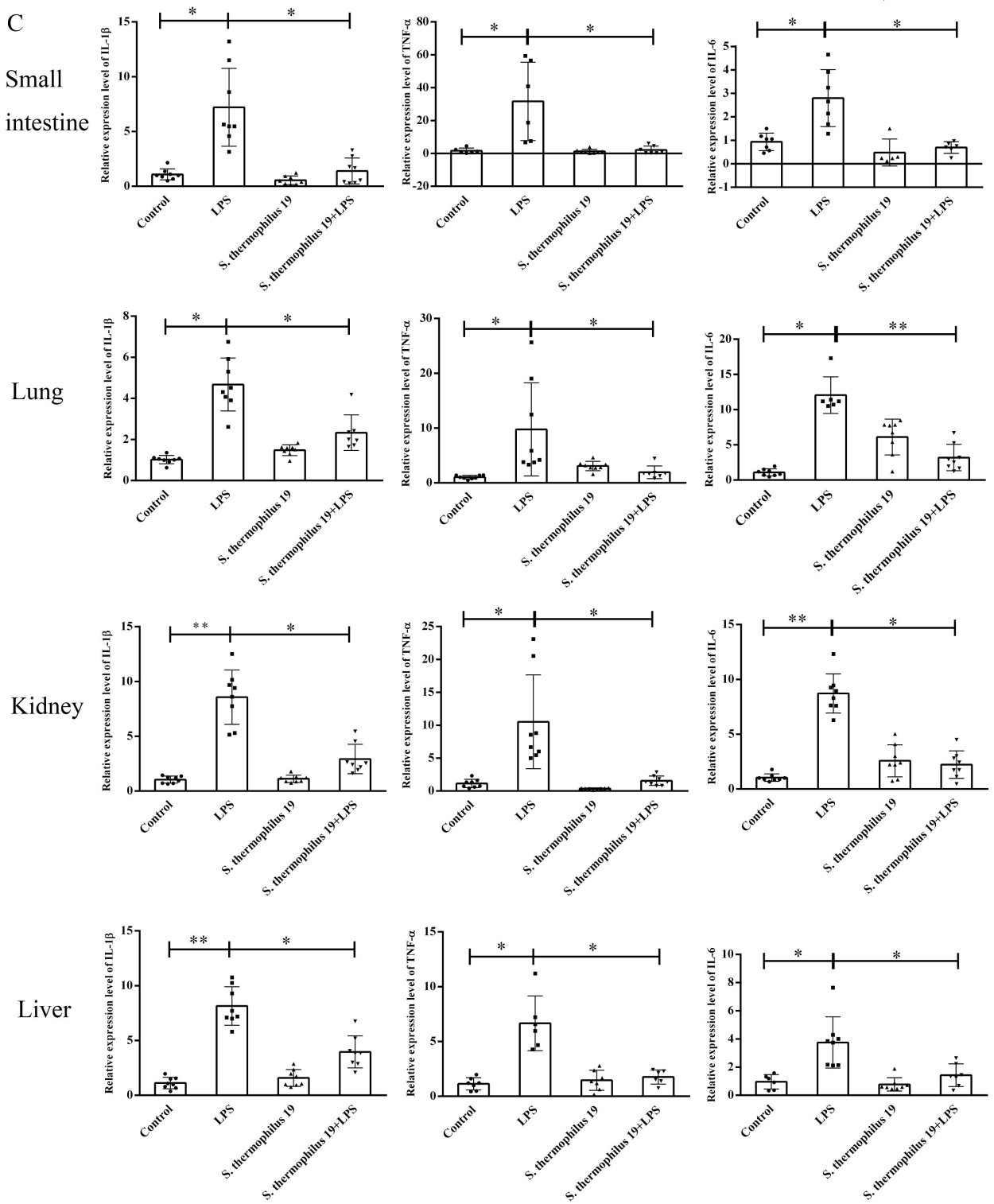

D

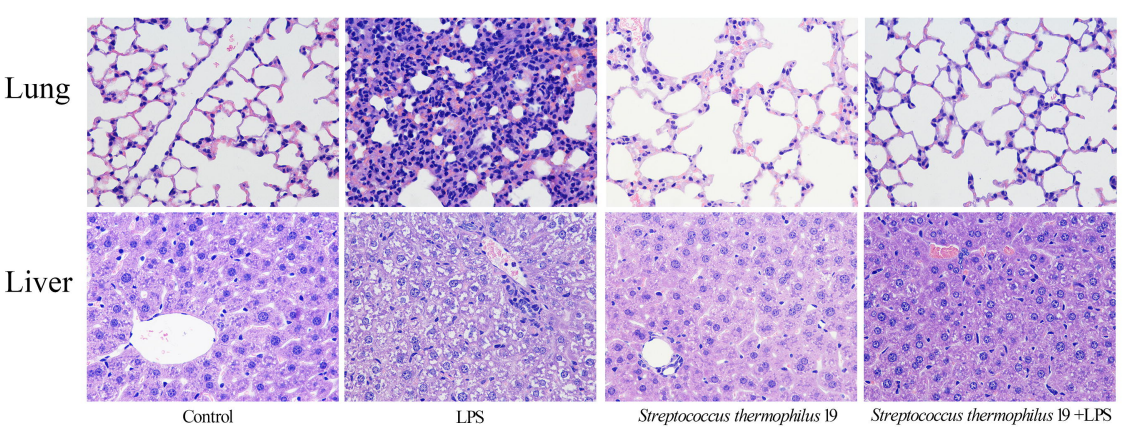


A

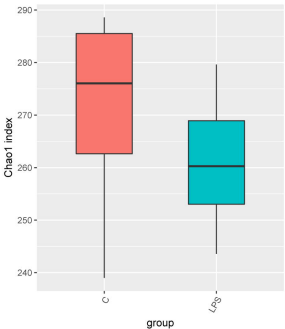

B

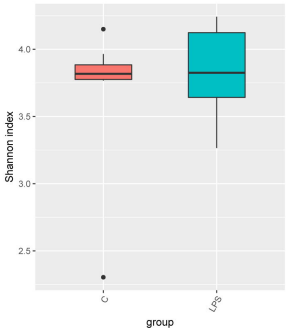

C PLS_DA

group

早

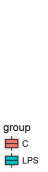

D

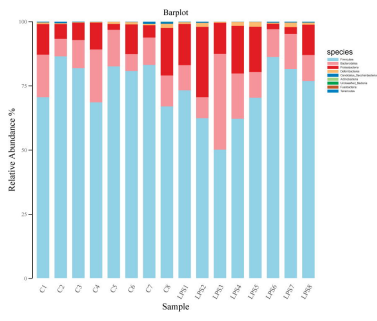


A ${ }^{200-}$

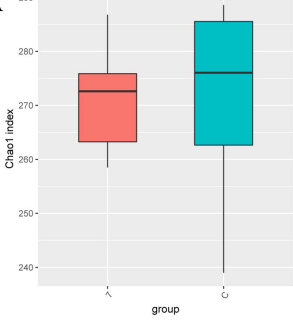

B
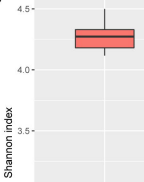

$30=$

2.5-

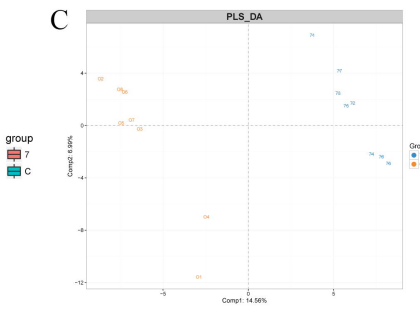

Group
$: 7$
0

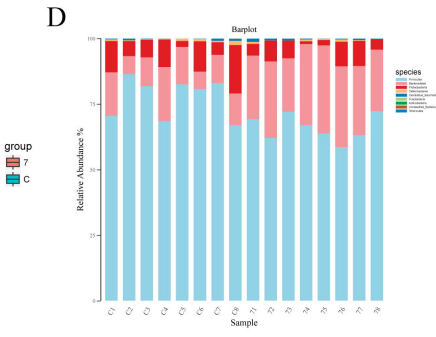



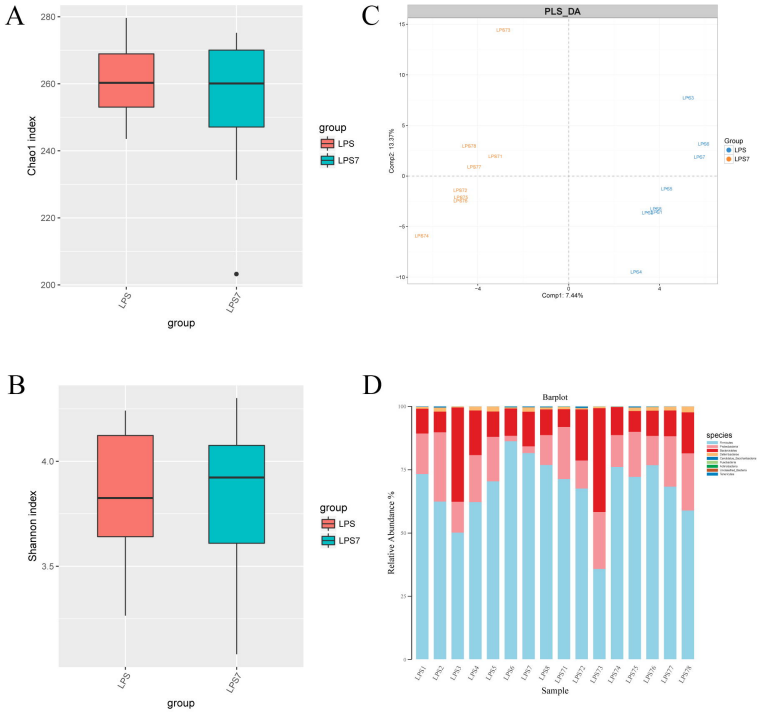


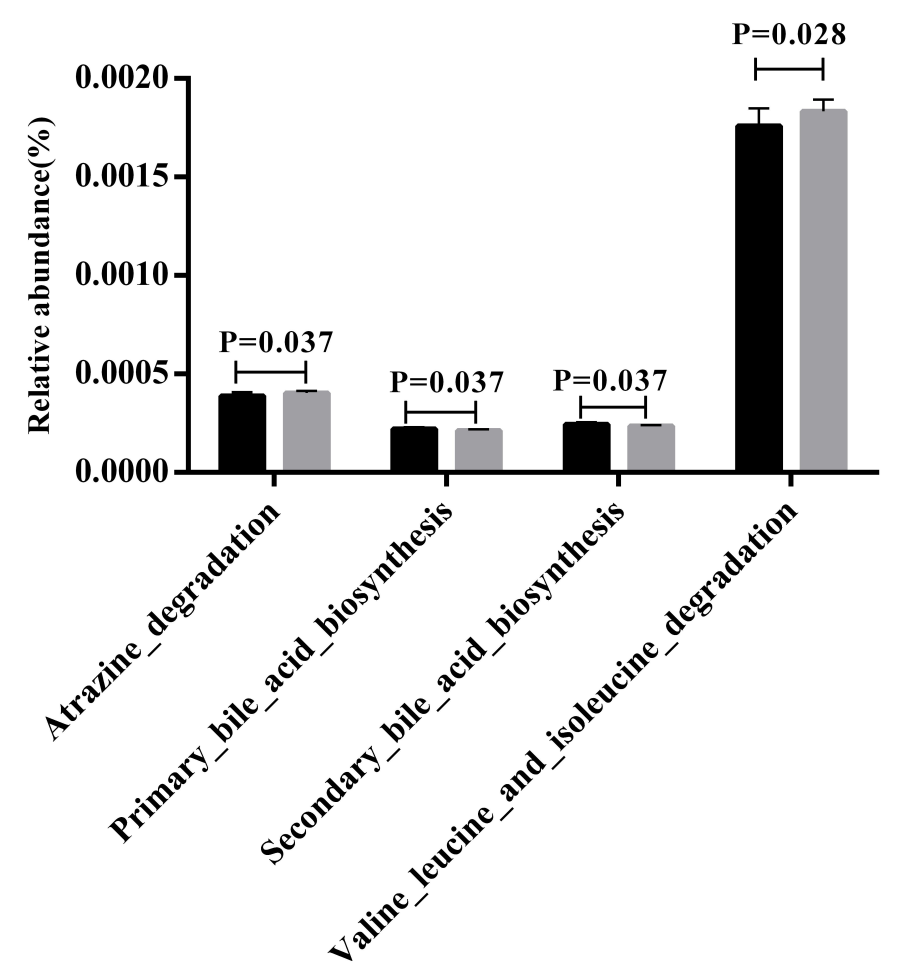

LPS

LPS+Streptococcus thermophilus 19 